\title{
PROJECTIVE AND IN JECTIVE OBJECTS IN THE CATEGORY OF BANACH SPACES
}

\section{KENNETH POTHOVEN}

Let $F$ denote either the field of real numbers or the field of complex numbers. Let $B$. be the category whose objects are Banach spaces over $F$ and whose morphisms are continuous linear maps $f: A \rightarrow B$ with norm $|f|=\sup _{|x| \leqq 1}|f(x)| \leqq 1$. The purpose of this note is to show that in $B$, without the restrictions (restrictions that make certain monomorphisms isometric (into) mappings and epimorphisms onto mappings) made on monomorphisms and epimorphisms in $[1],[3]$, and [5], the only injective object is the zero space and the only projective object is the zero space.

Notice that in $B$ a morphism $f: A \rightarrow B$ is a monomorphism if and only if it is one-to-one and is an epimorphism if and only if $f[A]$ $=\{b \in B \mid f(a)=b$ for some $a \in A\}$ is dense in $B$.

Lemмa 1. F is a retract of every nonzero Banach space.

Proof. Let $B$ be a nonzero Banach space in $B \cdot$. Fix $b_{0} \in B, b_{0} \neq 0$. Let $b_{0}^{*}: B \rightarrow F$ be a morphism with $\left|b_{0}^{*}\right|=1$ and $b_{0}^{*}\left(b_{0}\right)=\left|b_{0}\right|[2$, Corollary 14, p. 65]. Then $b_{0}^{*}\left(b_{0} /\left|b_{0}\right|\right)=1$. Define $f: F \rightarrow B$ by $f(m)=m b_{0} /\left|b_{0}\right|$. Then $f$ is a morphism in $B$. and $b_{0}^{*} \circ f=1_{F}$ (the identity morphism of $F$ ). Therefore $F$ is a retract of $B$.

LEMMA 2. $F$ is not injective in $B$.

Proof. Let $l_{1}$ be the Banach space consisting of sequences $a=\left(a_{i}\right)_{i \in N}, a_{i} \in F$ and $N=\{1,2, \cdots\}$, such that the norm $|a|=\sum_{i \in N}\left|a_{i}\right|<\infty$. Define $g: l_{1} \rightarrow l_{1}$ by $g\left(\left(a_{i}\right)_{i \in N}\right)=\left(a_{i} / i+1\right)_{i \in N}$. Then $g$ is a monomorphism in $B$. Let $b=(1 / i !)_{i \in N}$. Since $|b|=\sum_{i \in N} 1 / i !=e-1, b \in l_{1}$. Now let $b^{*}: l_{1} \rightarrow F$ be a linear function with $\left|b^{*}\right|=1$ and $b^{*}(b)=|b|$. There is no morphism $u: l_{1} \rightarrow F$ in $B$. such that $u \circ g=b^{*}$, i.e. so that the following diagram commutes:

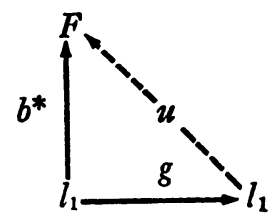

If such a morphism $u$ existed in $B$, then $(u \circ g)(b)$ must be $|b|$. However $g(b)=e-2<1$ but $|u(g(b))|=|b|>1$. This is a contradiction.

Received by the editors November 25, 1968. 
Lemma 3. $F$ is not projective in $B$.

Proof. Let $c_{0}$ be the Banach space consisting of sequences $a=\left(a_{i}\right)_{i \in N}, a_{i} \in F$, and $N=\{1,2, \cdots\}$, converging to zero with norm $|a|=\sup _{i \in N}\left|a_{i}\right|$. Define $g: c_{0} \rightarrow c_{0}$ by $g\left(\left(a_{i}\right)_{i \in N}\right)=\left(a_{i} / i\right)_{i \in N}$. Then $g$ is an epimorphism and a monomorphism in $B$. Let $b$ be the sequence $(0,2,0,0, \cdots)$ in $c_{0}$. Then $g(b)=(0,1,0,0, \cdots)$. Define $f: F \rightarrow c_{0}$ by $f(n)=(0, n, 0,0, \cdots)$. There does not exist any morphism $u: F \rightarrow c_{0}$ in $B$ such that gou $=f$, i.e. so that the following diagram commutes:

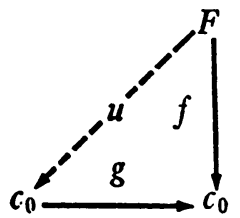

If such a morphism $u$ existed in $B$, then $u(1)$ must be $b$ as $g$ is one-toone. However then $|u|=\sup _{|a| \leqq 1}|u(a)| \geqq|u(1)|=2>1$. This is a contradiction.

Proposition 1. In $B$, if $B$ is injective, then $B=0$.

Proof. If for objects $A_{1}$ and $A_{2}$ in a category $C, A_{1}$ is a retract of $A_{2}$ and $A_{2}$ is injective, then $A_{1}$ is injective. The result follows immediately from Lemmas 1 and 2 .

Proposition 2. In $B$, if $B$ is projective, then $B=0$.

Proof. If in a category $C$ object $A_{1}$ is a retract of object $A_{2}$, and $A_{2}$ is projective, then $A_{1}$ is projective. The result follows from Lemmas 1 and 3 .

The author wishes to thank Dr. Kung-Wei Yang, Western Michigan University, for his guidance.

\section{REFERENCES}

1. H. B. Cohen, Injective envelopes of Banach spaces, Bull. Amer. Math. Soc. 70 (1964), 723-726.

2. N. Dunford and J. T. Schwartz, Linear operators, Part 1, Interscience, New York, 1958.

3. S. Eilenberg and J. C. Moore, Foundations of relative homological algebra, Mem. Amer. Math. Soc. No. 55 (1965).

4. J. R. Isbell, Injective envelopes of Banach spaces are rigidly attached, Bull. Amer. Math. Soc. 70 (1964), 727-729.

5. Z. Semadeni, Spaces of continuous functions on compact sets, Advances in Math. 1 (1958), 319-382.

Western Michigan University 